\title{
Defizite politischer Führungskraft und die Schwierigkeiten ihrer Überwindung
}

\section{Von der Notwendigkeit politischer Entscheidungsfindung}

Wilfried von Bredow

\begin{abstract}
Kernaussagen
Politische Führung ist ein knappes Gut in der deutschen Politik. Angesichts zahlreicher sich überlagernder Problemkomplexe ist ihre Abwesenheit besonders schmerzlich, da ausstehende Entscheidungen und Reformprogramme die Entscheidungsspielräume zukünftiger Generationen einengen. Diese Entscheidungen betreffen innenpolitische Fragestellungen ebenso wie die Rolle Deutschlands in der Welt. Bei der Formulierung von außenpolitischen Führungsansprüchen hält sich die Bundesregierung aus guten Gründen zurück. Dennoch ist sie dazu angehalten, ihrer Verantwortung nicht auszuweichen und politische Führungskraft zu entwickeln.
\end{abstract}

\section{Einleitung}

Auch in Deutschland ist politische Führungskraft, ganz besonders in Verbindung mit politischer Urteilskraft, eine nicht gerade im Überfluss vorhandene Ressource. Das erweist schon ein kurzer Rückblick auf die politische Geschichte. In unserem Land zählen Politiker wie Otto von Bismarck, Gustav Stresemann oder in der Nachkriegszeit Konrad Adenauer zu den Ausnahmegestalten. Die deutsche Geschichte des vorigen Jahrhunderts liefert im Übrigen auch ein Beispiel bösartiger politischer Führungskraft, personalisiert in der Figur Adolf Hitler. Aus diesem Beispiel kann man erkennen, dass zur Führungskraft in der Politik nicht nur macht-kalkulierende Urteilskraft gehören soll sondern auch eine Werte-Verbindlichkeit, welche die Traditionen politischer Moralität nicht zertrümmert sondern pflegt und womöglich behutsam weiterentwickelt.

Führungskraft ist in allen Organisationen gefragt, keinesfalls nur in der Politik, sondern z. B. auch in den Streitkräften, in der Wirtschaft oder in Kirchen und sozialen Bewegungen. Was dabei im einzelnen nötig ist und wie sie sich zeigt, variiert beträchtlich (vgl. Grasselt \& Korte, 2007). Nehmen wir die eingehende sehr ergiebige Fachliteratur in den Blick, fällt auf, dass insbesondere auch systematische Politikvergleiche anhand dieses Themas angestellt werden können (Sebaldt \& Gast, 2010; Gast, 2009; Helms, 2009). Im folgenden soll es indes in erster Linie um die deutsche Politik gehen, genauer: um die Politik der christdemokratisch-liberalen Regierungskoalition seit 2009. Bis heute bündelt sich die vielfältige und oft scharfe Kritik an dieser Regierung in dem Argument, es mangele ihr an politischer Führungskraft. Es versteht sich von selbst, dass der Hauptadressat solcher Kritik die Bundeskanzlerin ist.

Konzentrieren wir uns auf die politische Führung an der Spitze eines Staates wie Deutschland, dann geht es dabei um folgendes:

- die Richtungsbestimmung der Willens- und Entscheidungsprozesse in der Regierung,

- die Kontrolle der Durchsetzung von getroffenen Entscheidungen durch den Regierungsapparat und die weiteren Behörden,

- das erfolgreiche Geltendmachen nationaler Interessen in den Außenbeziehungen,

- die Herstellung und Pflege der öffentlichen Zustimmung (politischen Akzeptanz) von Regierungshandeln.

Politische Führungskraft bemisst sich daran, ob und wie die Staatsspitze auf diesen vier Ebenen politische Führung zeigt. Dass sie dazu eine Reihe möglichst reibungslos funktionierender Instrumente braucht (Zuarbeit im Bundeskanzleramt, externe Beratung, belastungsfähige Vernetzung mit den Handlungseliten im Parlament und in der Gesellschaft), sei am Rande erwähnt. Aber gerade der Auf- und Ausbau solcher organisatorischen und personellen Hilfsmittel ist ein Zeichen von politischer Führungskraft. Mit Recht gilt das Adjektiv ,beratungsresistent' heute als vernichtendes Urteil über die Führungskraft eines Politikers oder einer Politikerin. Unter handwerklichen Gesichtspunkten gehören der antizipierende Scharfblick für die politische Bedeutung eines Themas, die Fähigkeit, Aufgaben zu delegieren, und die Bereitschaft, externe Expertise qua Politikberatung für das eigene Reden und Handeln nutzbar zu machen, zu den wichtigsten Voraussetzungen politischer Führungskraft.

Die folgenden Ausführungen sollen als ein Plädoyer für politische Führungskraft verstanden werden, nicht nur unter allgemeinen Gesichtspunkten sondern auch im Blick auf die gegenwärtige Bundesregierung. Deren Wahrnehmung in der Öffentlichkeit und besonders in einigen Medien hierzulande ist, nachdem sie im Herbst 2009 die Regierungsgeschäfte übernommen hat, nicht durchgehend von Zustimmung gekennzeichnet, milde gesagt. Nach dem Wahlsieg von CDU/ CSU und FDP und der schwarz-gelben Koalitionsbildung scheint es der Bundesregierung unter Kanzlerin Angela Merkel auf spektakuläre Weise an politischer Führungskraft zu fehlen. 
Illustrationen dafür gibt genügend. Die Klagen darüber, vor allem wenn sie aus dem Lager der Oppositionsparteien kommen, umweht allerdings ein kräftiger Hauch von Hypokrisie. Die unbefriedigende Erledigung der Regierungsgeschäfte hat ihnen schließlich bald nach den Wahlen erheblich bessere Umfragedaten eingebracht. Die Führungsblockaden im Regierungslager resultieren gewiss zu nicht geringen Teilen aus dem koalitionsinternen Streit. Jedoch darf auch nicht ganz übersehen werden, dass eine nicht unbeträchtliche Zahl der Schwierigkeiten, mit denen die Bundesregierung seit 2009 zu kämpfen hat, in den Sachproblemen selbst liegt. Anhand ausgewählter Beispiele, vor allem aus dem Bereich der Außen- und Sicherheitspolitik, sollen Gründe für das Entstehen von Führungsblockaden und Möglichkeiten zu ihrer Überwindung überdacht werden.

\section{Politische Führung und politische Führungskraft}

Politische Führung ist ein strategisches Konzept der Richtungsvorgabe, in dem kurz-, mittel- und langfristiges Handeln verbunden werden. Der Anspruch an die Person des Kanzlers oder der Kanzlerin, über ein solches Vermögen zu verfügen, kommt übrigens auch in einem wichtigen Begriff aus dem Grundgesetz zum Ausdruck (vgl. Schuett-Wetschky, 2008). Der erste Satz von Art. 65 GG lautet: „Der Bundeskanzler bestimmt die Richtlinien der Politik und trägt dafür die Verantwortung."

Ein politisches Gemeinwesen, jede politische Organisation, ganz unabhängig von ihrer Größe und ihren Zielen, kommt ohne politische Führung nicht aus. Das ist, wenn man so will, eine soziologische Beobachtung der Strukturen und des Funktionierens von Organisationen. Die Frage ist freilich immer, ob und in welchem Maße die politische Führungskraft der in den Organisationen handelnden Personen dieser Anforderung gerecht wird.

\subsection{Horizonte}

Politische Führung an der Spitze eines Staates muss sich vor vier Horizonten bewähren:

- dem politischen System im engeren (Regierung, Parlament) und weiteren Sinn (Partei, mediale Öffentlichkeit) zwecks Pflege der eigenen Mehrheit;

- bei der Formulierung von Konzepten und Programmen zwecks Durchsetzung nationaler Interessen in der Innenund der Außenpolitik;

- auf der Ebene europäischer Politik zwecks Mitgestaltung der im Rahmen der Europäischen Union zu treffenden Entscheidungen (Kommission, Europäischer Rat, Europäisches Parlament);

- auf weltpolitischer Ebene in zahlreichen internationalen Organisationen und Regimes zwecks Mitgestaltung einer globalen Ordnungspolitik.

In den letzten Jahrzehnten (Stichwort: Globalisierung) sind diese vier Horizonte näher aneinander gerückt und haben angefangen sich gegenseitig zu überlagern. Darüber hinaus ist es heute für einen Staat wie Deutschland unabdingbar, überall gleichzeitig sozusagen mit voller Kraft politische Führung zeigen zu können.

Deshalb stimme ich, bei allem Respekt für sehr viele seiner politischen Urteile, in diesem Punkte nicht mit Altkanzler Helmut Schmidt (2010) überein, der kürzlich in seiner Dankrede für die Verleihung des Point-Alpha-Preises formulierte: „Unser Feld ist nicht die Weltpolitik und nicht die atomare globale Strategie, nicht Asien, nicht der Nahe und Mittlere Osten oder Afrika, sondern unsere europäischen Nachbarn sind unser Arbeitsfeld. “ Gewiss gibt es für die deutsche Politik näher und ferner liegende Aufgaben. Jedoch ein programmatischer Verzicht auf Mitgestaltung der Weltpolitik, das kann keine sinnvolle Option für Deutschland sein.

\subsection{Führung und Demokratie}

Zwischen diesen beiden Begriffen gibt es eine Art Spannungsverhältnis, jedenfalls dann, wenn man sie beide konzeptionell strapaziert. Dann steht Demokratie für einen Prozess des Aushandelns von Kompromissen und der bottom-up-Entscheidung durch die Mehrheit, in dem politische Führung gewissermaßen abgeblockt wird. Denn mit dem Führungsbegriff werden autoritäre top-down-Entscheidungen assoziiert, die selbst dann, wenn sie innerhalb eines demokratischen Legitimationsrahmens erfolgen, doch vielfach als undemokratischer Stil angesehen werden. In der Demokratie muss politische Führung das Geschick besitzen, den politischen Diskurs so zu beeinflussen, dass die getroffenen Entscheidungen zumindest mittelfristig mehrheitsfähig bleiben. Darunter ist zu verstehen, dass sie von der Mehrheit entweder unterstützt oder hingenommen werden.

Eine ähnliche Zwiespältigkeit ergibt sich, wenn man nach der Qualität politischer Führungs-Entscheidungen in einer Demokratie fragt. Sollen sie eher innovativ sein und damit womöglich gegen eine Innovationen scheuende Mehrheit durchgesetzt werden? Oder doch eher dem erkennbaren Mehrheitswillen folgen, also responsiv? Das ist unter Politikern wie Politologen umstritten (vgl. Helms, 2010; Ritzi \& Schaal, 2010). Die Wiederbewaffnung der Bundesrepublik Deutschland wurde von Konrad Adenauer in der ersten Hälfte der 1950er Jahre gegen den Willen der Mehrheit unter den Bundesbürgern durchgesetzt. Das war eindeutig ein Ergebnis starker politischer Führungskraft - aber für viele Gegner der Wiederbewaffnung galt diese Entscheidung damals als undemokratisch und demokratiegefährdend.

\subsection{Führung und Kommunikation}

Politische Führung braucht politische Kommunikation, und zwar nach innen, mit anderen politischen Handlungsträgern im eigenen Land und in anderen Ländern, mit der Öffentlichkeit im eigenen Land und in anderen Ländern (einschließlich der allerdings weitgehend fiktiven „Weltöffentlichkeit“). 
Im Blick auf die Verhältnisse bei uns (in manchen anderen westlichen Demokratien ist das so ähnlich) fallen vor allem drei Aspekte politischer Kommunikation ins Auge: die Führung von Wahlkämpfen, die Abstimmung in der Koalition sowie das sogenannte media management.

Wahlkämpfe sind heute zu einem Feld hoch-professioneller Werbeagenturen und politischer spin-Doktoren geworden. Rückgängig machen lässt sich das nicht, obwohl es auch etwas Widerliches hat (ganz besonders etwa die Schmutzkampagnen und andere Taktiken unterhalb der Linie politischer Anständigkeit). In Wahlkämpfen werden in aller Regel die jeweils eigenen Führungsqualitäten übertrieben, so dass inzwischen viele Wähler schon gar nicht mehr enttäuscht sind, wenn die Wahlversprechen nicht oder nur teilweise durchgesetzt werden können. Viele Wähler indes sind in der Tat enttäuscht und verbittert und werden so leicht Opfer ihrer eigenen Verdrossenheit oder fallen auf noch weit kräftiger übertriebene Wahlversprechen herein.

Abstimmung in der Koalition (bei uns) oder über die Parteigrenzen hinweg (z. B. in den USA) sind nötig, um bestimmte Entscheidungen zu fixieren und parlamentarisch durchzusetzen. Die jetzige Regierungskoalition war mit ihrer internen Kommunikation bislang wenig erfolgreich, wobei - für manche überraschend - insbesondere die CSU mit ihrem angeschlagenen regionalen Sendungsbewusstsein ein einheitliches Erscheinungsbild der Koalition immer wieder in Frage stellt.

Politische Kommunikation erreicht die Öffentlichkeit nur in den wenigsten Fällen direkt sondern über die Medien. Die Medien sind aber viel mehr als nur Vermittler, Lautverstärker von Regierungskommunikation. Das ist auch, grundsätzlich jedenfalls, gut so. Der Sammelbegriff Medien umfasst verschiedene technische Medien (Print-Medien, Rundfunk, Fernsehen, Internet), deren Wirkung auf die Öffentlichkeit auch ganz unterschiedlich ist. Er umfasst auch Medien innerhalb der gleichen Kategorie mit sehr unterschiedlichem politischem Selbstverständnis und politischem Ethos. Deswegen ist es immer ein bisschen prekär, wenn man generalisierend von „den“ Medien spricht (vgl. Meyer, 2001). Es gehört zu den Grundlagen einer funktionierenden westlichen Demokratie, dass in den Medien eine große Meinungsvielfalt herrscht. Auch soll die Regierungskommunikation in den Medien nicht kritiklos übernommen werden.

Die Sichtbarkeit politischer Führungskraft basiert nicht zuletzt auf der Kommunikation ihrer Ergebnisse. Kritiker dieses Sachverhalts gehen so weit, von einer „Mediendemokratie“ oder „Mediokratie“ zu sprechen. Sie meinen damit, dass vergleichsweise mehr Anstrengungen gemacht werden, politische Führung als kraftvoll erscheinen zu lassen als sie tatsächlich ist. Plebiszitäre oder „schein-plebiszitäre“ Politikformen oder ein medienwirksamer, den Meinungsmachern in manchen Medien geradezu hechelnd nachlaufender Populismus verdrängen so innovative, aber der Öffentlichkeit schwer zu vermittelnde Entscheidungen. Wie es scheint, tragen seit ein paar Jahren vor allem politische Kommunikationsformen des Internets zu einer drastischen Vergröberung politischen Argumentierens bei. Die oft genug hanebüchen beschränkten und vorurteils-belasteten, zugleich meist in salopper bis grob beleidigender Sprache hingeschrieben Reaktionen von Lesern auf Artikel in den Online-Ausgaben auch professionell anspruchsvoller Qualitäts- Zeitungen werden auf die Dauer, so steht zu befürchten, auf die Arbeit der Journalisten eine vergröbernde Wirkung ausüben.

Als Zwischenfazit lässt sich festhalten, dass zu Zeiten eines erhöhten politischen Problemdrucks politische Führung im Sinne von durchschlagenden Problemlösungen schwieriger geworden ist: mehr Verantwortlichkeit und größere Erwartungen auf der einen, geringere Gestaltungsmöglichkeiten auf der anderen Seite.

\section{Berliner Führungsprobleme}

„Seit ihrem Regierungsantritt legt die Truppe um Angela Merkel einen selbst erklärten Neuanfang nach dem anderen hin. Bisher sind noch alle fehlgeschlagen. „Klappt es diesmal? “ Das fragte die Spiegel-Redaktion maliziös die Leser ihrer Online-Ausgabe vom 1. Juli 2010 nach der mühevoll über die Bühne gegangenen Wahl von Christian Wulff zum Bundespräsidenten. Die Aussage ist unzweideutig: Angela Merkel hat ihr Kabinett (die „Truppe“) nicht zu einem leistungsorientierten Team formen können. Der interne Streit in der Regierung und innerhalb der Koalitionsparteien über die Ausrichtung der Regierungspolitik lähmt sie. Die erst im dritten Wahlgang erreichte Mehrheit für den Kandidaten des Regierungslagers und die aus diesem Lager herausgebrochenen Stimmen für den gemeinsam von SPD und GRÜNEN präsentierten Oppositionskandidaten Gauck wurden nicht nur von der regierungskritischen Presse als Quittung („,Denkzettel“) für enttäuschte Erwartungen an die Adresse der Bundeskanzlerin interpretiert.

Es ist bezeichnend für die Kakophonie im Regierungslager, die gleich nach dem Wahlsieg im September 2009 einsetzte, dass dabei wie ein Leitmotiv immer wieder die Aufforderung an Angela Merkel erhoben wird, nun endlich einmal politische Führungskraft zu zeigen, ein Machtwort zu sprechen. Zugleich mit dieser Forderung werden aber inhaltlich und stilistisch heftige Attacken gegen die anderen Parteien in der eigenen Koalition oder gegen bestimmte Minister geritten. Von außen betrachtet, wie von innen auch: ein Trauerspiel.

Warum ist das so? Warum verlängert sich ein Zustand, den alle Beteiligten als beklagenswert ansehen und von dem sie wissen, dass sie ihn im Interesse des Landes, das sie regieren, aber auch in ihrem eigenen Interesse, so schnell wie möglich abstellen müssen?

Die Probleme, Schwierigkeiten und Defizite hinter diesen und ähnlichen Fragen lassen sich auf vier Ebenen verorten:

- auf der personellen Ebene - vor allem, wenn man vermutet, dass das für staatliche Führungsaufgaben eingesetzte (gewählte) Personal „es nicht kann“, um die flapsige Bemerkung eines Politikers im Ruhestand aufzugreifen; 
- auf der Ebene der Koalition - vor allem, wenn erkennbar wird, dass die in der Regierung programmatisch kooperierenden Parteien nicht zu einer fruchtbaren und erfolgreichen Zusammenarbeit finden können, weil sie zu wenig gemeinsame Ziele verfolgen;

- auf der innergesellschaftlichen Ebene - vor allem, wenn die unterschiedlichen gesellschaftlichen Interessen und politischen Perzeptionen sich gegenseitig in die Quere kommen und auf diese Weise keine Reformen verwirklicht werden können, weil sich sogleich heftiger Widerstand gegen sie aufbaut, obwohl ein großer (aber eben nie derselbe) Teil der Gesellschaft mit dem Ausbleiben von Reformen und Veränderungen zunehmend unzufrieden ist;

- schließlich auf der internationalen Ebene - vor allem, wenn die Staaten wegen der jeweiligen internen Druckverhältnisse auf ihre Regierungen zu gemeinsamen Handeln sich selbst dort nicht entschließen können, wo das Unterlassen von Veränderungen in wenigen Jahren zu großen, wenn auch im einzelnen schwer zu ermessenden Problemen für sie alle führen wird.

Im politischen Alltag sind diese vier Ebenen aufs Engste miteinander verschränkt. Die Wirkung dieser Faktoren kommt besonders spektakulär zur Geltung, wenn unvorhergesehene Ereignisse in der Innen- oder Außenpolitik ein rasches gemeinsames Handeln aufgrund gleichartiger Lagebeurteilungen nötig machen. Weil es meistens mehrere Gründe für erkannte Führungsmängel gibt, nützen einfache Heilmittel zu ihrer Überwindung nichts, wenn sie nur auf einer Ebene wirksam sind.

\subsection{Kanzler-Rückblicke}

Im Rückblick auf das Führungsverhalten der verschiedenen Bundeskanzler seit 1949 ergibt sich bald der Eindruck, dass sie mit wenigen Ausnahmen sehr häufig in der öffentlichen Kritik standen, weil sie entweder zu wenig Führungskraft demonstrierten oder, dann aber nicht immer an der richtigen Stelle, zu viel. Konrad Adenauer kommt da vergleichsweise noch mit am besten weg, weil er seine zuweilen widerspenstigen Minister, Koalitionspartner oder Mitglieder der eigenen Fraktion mit List und Geschick „auf Vordermann“ zu bringen verstand. Sein Nachfolger Ludwig Erhard hatte es als Kanzler diesbezüglich sehr viel schwerer; im Volksmund (auch dem „höheren“) wurde er, vor allem in direkten Vergleichen mit seinem Vorgänger, häufig als „Gummilöwe“ bezeichnet. Kurt-Georg Kiesinger stand der ersten Großen Koalition als Kanzler vor, in seinem Kabinett waren eine Reihe starker Persönlichkeiten, und eine herausgehobene Rolle für die Führung der Regierungsgeschäfte spielten auch die Vorsitzenden der Bundestags-Fraktionen von CDU/CSU (Rainer Barzel) und SPD (Helmut Schmidt). Der Kanzler selbst galt als nicht besonders führungsstark, vielmehr als „wandelnder Vermittlungsausschuss“ zwischen den starken Persönlichkeiten in seiner Regierung.
Auch Willy Brandt ließ seinen Ministern die Zügel lang, er führte sozusagen mit dem Mittel der großen Vision. Ganz anders sein Nachfolger: Helmut Schmidt gilt als besonders führungsstarker Kanzler, der bei Widerspruch rasch ungeduldig aufbrauste. Aber erstens übertrieb er es damit zuweilen, gerade auch in der internationalen Politik. Und zweitens vermochte er in wichtigen Fragen weder den Koalitionspartner (in Wirtschaftsfragen) noch seine eigene Partei (in Sicherheitsfragen) hinter sich zu bringen. Helmut Kohls Führungsstärke zeigte sich vor allem in zwei Dingen, in dem MikroManagement seiner eigenen Partei und in seiner vielzitierten Beharrlichkeit bei bestimmten Grundsatzfragen. Unter tätiger Mithilfe des amerikanischen Präsidenten und einiger seiner Berater ergriff er 1989 einen Zipfel des Mantels der Geschichte und sicherte sich damit einen Platz in den Geschichtsbüchern als entscheidungskräftiger VereinigungsKanzler, obwohl er auf vielen anderen Politikfeldern vor und nach 1990 viele Führungsprobleme hatte. Manche davon überwand er durch „Aussitzen“. Kohls Nachfolger Schröder hat den Ruf eines führungsstarken „Basta!“-Kanzlers - aber manche dieser zuweilen etwas selbstherrlich präsentierten Entscheidungen haben auch, besonders in der Außenpolitik, Sand ins Getriebe geblasen.

\subsection{Angela Merkels Führungsstil}

Eigentlich kann man nicht sagen, dass Angela Merkel „umstritten“ ist, so wie es andere Politiker waren und sind, die durch rasche Entscheidungen und mehr noch durch ihre Rhetorik in der Öffentlichkeit polarisierend wirken. Für jenen Typ Politiker steht nach wie vor Franz Joseph Strauß als Prototyp; von den Jüngeren ist hier vor allem etwa Roland Koch zu nennen. Dass Angela Merkel rasch und im Notfall auch beinhart entscheiden kann, wird eher anhand ihres Aufstiegs innerhalb der CDU deutlich. Als Kanzlerin führt sie, um den jetzigen Bundespräsidenten zu zitieren, „von hinten“. Das habe, so meint der Journalist Matthias Geis in durchaus kritischer Absicht, zu einer Koalition geführt, in der bei allem Konflikt die sachorientierte Verantwortungsbereitschaft der Beteiligten dominierte. „Doch nun hat es die Kanzlerin mit egozentrischen Partnern zu tun, die zwischen triumphaler Selbstüberschätzung und tief sitzenden Minderwertigkeitskomplexen zu schwanken scheinen. Ihnen eröffnet der tolerante, beobachtende Führungsstil erst den Raum, den sie für ihre oft ziellosen Profilierungskämpfe nutzen“ (Geis, 2010). Das Fazit des Autors lautet entsprechend, Angela Merkel müsse ihre Art der Führung verändern, wenn sie ihre Führung verteidigen wolle.

Ein Stück weit kann man dieser Analyse folgen, wenngleich sie wegen der Verengung des Horizonts auf den Führungsstil unter Auslassung aller politischen Inhalte den Sachverhalt zu stark verkürzt.

Die Atmosphäre in der schwarz-gelben Koalition ist allerdings in der Tat nebliger, als sie es zwischen 2005 und 2009 in der Großen Koalition war, und das lag nicht ausschließlich, indes weitgehend an den beiden kleinen Koalitionspart- 
nern CSU und FDP. Das strukturelle Problem der CSU besteht dabei vor allem darin, ihr bundespolitisches Wirken so darzustellen, dass es in Bayern als optimale Verstärkung der Landespolitik wahrgenommen wird. Das ist kein neues Problem der CSU in ihrem Verhältnis zur CDU. Aber es ist jüngst wegen des schleichenden Verlusts der lange unangefochtenen politischen Hegemonie der CSU in Bayern erheblich dringlicher geworden und deshalb für einige leicht panische Aktionen von CSU-Politikern verantwortlich. Das Problem der FDP besteht in ihrer dünnen Personaldecke an der Spitze und der Verkümmerung ihrer politischen Programmatik. Beide Probleme lassen sich nicht einfach durch Führungs-Entscheidungen an der Regierungsspitze aus der Welt schaffen. Es ist ja mehrfach versucht worden, Stichwort „Neustart“, aber absehbar ohne durchschlagenden Erfolg.

\subsection{Die Aufgaben der Regierungskoalition}

Die großen „Baustellen“, wie eine gängige Metapher lautet, der Bundesregierung sind allesamt besonders komplex und verlangen einen beträchtlichen Aufwand an politischer Kreativität.

- Die demographische Entwicklung und bestimmte Veränderungen in der Arbeitswelt machen eine Veränderung des Alterssicherungssystems notwendig. Je länger mit einer Rentenreform gewartet wird, desto tiefer werden die unumgänglichen Maßnahmen in das bestehende System einschneiden.

- Auch das Gesundheitssystem kann nicht länger so fortgeschrieben werden, weil es demnächst nicht mehr finanzierbar sein wird, wenn es nicht gründlich reformiert wird.

- Für die langfristige Sicherung der Energieversorgung braucht es ein Konzept, das Kosten-Gesichtspunkte mit solchen der Verminderung von Importabhängigkeit und vor allem auch der Nachhaltigkeit und der Umweltverträglichkeit kombiniert. Die Versuche der Regierung, über eine Verlängerung der Laufzeiten deutscher Atomreaktoren Zeit zu gewinnen und den Anstieg der unmittelbaren Energiekosten zu bremsen, ist durch die ReaktorKatastrophe in Japan im März 2011 und seine Wahrnehmung in der deutschen Öffentlichkeit gescheitert.

- Die internationale Sicherheits-Landschaft hat sich nach dem Ende des Ost-West-Konflikts dramatisch verändert. Alte Bedrohungsszenarien sind ins Archiv gewandert; dafür gibt es neue, auf die sich vorzubereiten unter anderem eine weitgehende Reform der Streitkräfte voraussetzt. Weil die Anforderungen an die Bundeswehr heute ganz anders als früher sind, müssen ihre Strukturen, ihre Bewaffnung und Ausrüstung, müssen die Ausbildung und das professionelle Profil der Soldaten verändert werden.

- In der Aufräum-Phase zur großen Finanzkrise 2008/09 müssen die Grundparameter im Verhältnis Staat/Wirtschaft neu justiert werden. Als weitere Folge dieser Krise ist es dringlicher geworden, die Staatsschulden abzubauen, was erheblicher Kraftakte bedarf, denn das öffentliche Sparen ist nur abstrakt und solange es einen nicht selbst betrifft, populär.
- Die Auswirkungen der Finanzkrise auf die europäische Integration sind dramatisch. Die Notwendigkeit, den Euro zu stützen und hoch-verschuldete Länder wie Griechenland, Irland und Portugal gegen Abwertungs-Spekulationen zu schützen, verlangt insbesondere von Deutschland als dem wirtschaftlich stärksten EU-Mitglied beträchtliche Opfer.

Diese Liste ist längst nicht vollständig. Bestimmte Schlüsselworte der politischen Debatte wie Bildung, Integration der Zuwanderer, Klimawandel tauchen in ihr noch gar nicht auf. Aber was ich mit der Liste zum Ausdruck bringen will, ist klar geworden: Diese Regierung hat es mit einer sehr großen Zahl sehr schwieriger Herausforderungen zu tun. Das soll Führungsmängel nicht entschuldigen, aber doch ein wenig Verständnis dafür wecken, dass die Vorstellung (performance) der Bundesregierung im ersten Jahr nach dem Wahlsieg nicht so überzeugend ausgefallen ist.

\subsection{Beispiel Bundeswehr-Reform}

Die Reformbereitschaft des im Herbst 2009 neu berufenen Verteidigungsministers zu Guttenberg und die Art und Weise, wie er sie nach innen und außen demonstriert hat, war für viele Beobachter durchaus beeindruckend. Er hat als einer der ersten die fatale Beschönigungs-Rhetorik bezüglich des ISAF-Einsatzes der Bundeswehr aufgebrochen, die sich in mehreren Jahren wie ein geistiger Mehltau über die Wahrnehmung der Vorgänge in Afghanistan gelegt hatte (von Bredow, 2010). Und wenn am Ende tatsächlich eine ReformVorstellung für die Bundeswehr beschlossen und zügig umgesetzt wird, wie sie das Modell Vier seiner Alternativvorlage vorsieht, dann wird man später in den Geschichtsdarstellungen über die Bundeswehr viel von der Führungsstärke dieses Ministers lesen können. Der schmähliche Rücktritt des Ministers Anfang März 2011 wegen einer Plagiatsaffäre verdüstert den Eindruck, den der Minister auf große Teile der Öffentlichkeit gemacht hat. Seinem Amtsnachfolger Thomas de Maizière werden die Dynamik und Führungskraft zu Guttenbergs nicht zugetraut. Er steht insofern eher in der Tradition von Guttenbergs Vorgängern, den Ministern Jung (CDU), Struck (SPD) und Scharping (SPD). Sie alle haben sich mehr oder weniger mit der Reform der Bundeswehr abgemüht und durch Kommissionen oder mit anderen Instrumenten vorbereitete Reform-Beschlüsse gefasst. Aber sie haben um einen grundlegenden Aspekt dieser Reform einen politischen Bogen gemacht, nämlich um die Einführung der Freiwilligen-Streitkräfte.

Die Diskussion um die Allgemeine Wehrpflicht zeichnet sich in Deutschland durch eine besondere Zähigkeit aus. Am 20. Juli 2009 meinte Bundeskanzlerin Merkel beim Feierlichen Gelöbnis vor dem Reichstag: „Die Wehrpflicht ist zum Markenzeichen unserer Streitkräfte geworden, um das wir auch international beneidet werden. “ Das ist so einer von den Politiker-Sätzen, über die man ins Grübeln gerät, bevor man bemerkt, dass es sich nicht lohnt. Schließlich ist der An- 
teil der Wehrpflichtigen am Umfang der Streitkräfte nur sehr gering; für Auslandseinsätze stehen sie nur als Freiwillige zur Verfügung; von einer engen Verbindung zwischen ziviler Gesellschaft und Bundeswehr wegen der Aufrechterhaltung der Wehrpflicht kann keine Rede sein; eine Sechs-Monate-Wehrpflicht ist kaum mehr als ein verlängertes Praktikum; das Leitbild des Staatsbürgers in Uniform und die Innere Führung sind bei der Aussetzung der Wehrpflicht keineswegs in Frage gestellt; Rekrutierungsprobleme gibt es auch mit der Wehrpflicht usw. Kurz: das Profil der sicherheitspolitischen Herausforderungen Deutschlands und seiner Streitkräfte verlangt spätestens seit dem BVerfG-Urteil vom Sommer 1994 eine Professionalisierung der Bundeswehr, um mehr Soldaten für Einsätze in internationalen Friedens- und Stabilisierungsmissionen der verschiedensten Art bereitstellen zu können.

Diese relativ simple Einsicht prallte indes jahrelang an einer aus den seltsamsten Argumenten bestehenden Mauer ab. Dahinter stand eine wirklich Große Koalition von Regierung und Parlament (Ausnahmen: FDP und die GRÜNEN), Sicherheits- und Sozialpolitikern, Interessenverbänden (einschließlich des meistens sehr sachangemessen argumentierenden Bundeswehrverbandes) und Soldaten in obersten Diensträngen. Von letzteren änderte ein Teil zwar seine Meinung nach der Pensionierung, aber das brachte dann auch nichts mehr ein.

Die erst jetzt, nach den Erfahrungen des bewaffneten Kampfes in Afghanistan und der sich langsam anbahnenden öffentlichen Debatte über die politische Bedeutung solcher Einsätze, sich gegen hinhaltenden Widerstand vieler CDUund CSU-Politiker durchsetzende Erkenntnis von der Unabdingbarkeit von Freiwilligen-Streitkräften ist ein Beispiel für die Reform-Schwerfälligkeit der deutschen Politik. Wenn man dieser Schwerfälligkeit aber nicht ein wenig Beine zu machen versteht, dann addieren sich Führungsschwäche und das Spielen auf Zeit zu folgenreichen Führungsmängeln zum Schaden einer zukunftsorientierten Politik.

\subsection{Nationales Interesse und internationale Ordnung}

Nur eine solche liegt im nationalen Interesse. Mit diesem Begriff ist selbstverständlich kein „right or wrong - my country" gemeint, und es drückt auch nicht irgendein vergangenheitsdurchtränktes Hegemoniestreben im Verhältnis zu den Nachbarstaaten aus.

Deutschland als die Zentralmacht Europas hat in seiner Außenpolitik multilaterale Kooperation zu seiner Hauptmethode gemacht und ist dabei gut gefahren, was sich im Prozess der Wiedervereinigung 1989/90 gezeigt hat. In Europa, genauer in der Europäischen Union, ist die bilaterale Zusammenarbeit mit Frankreich besonders wichtig. Aber damit diese sinnvoll funktioniert, nämlich so, dass die anderen EUMitglieder auf die Integrationsreise mitgenommen und nicht etwa vor den Kopf gestoßen werden, bedarf es einer besonders umsichtigen deutschen Führung. Antonio Puri Purini (2010), von 2005 bis 2009 Botschafter Italiens in Berlin, hat kürzlich geschrieben, in der Europapolitik sei nun die Stunde der Angela Merkel gekommen. Er begründet diese Feststellung - halb Anspruch, halb Hoffnung - folgendermaßen: „Als Teil einer europäischen Gemeinschaft, mit der sie seit ihrer Gründung im Jahr 1949 schicksalhaft verbunden ist, und als wirtschaftlicher und politischer Motor Europas hat die Bundesrepublik die Pflicht, sich für eine Wirtschaftsunion stark zu machen, die weit über die gemeinsame Währung hinausreicht. Sie muss bekräftigen, dass die...Stabilität ein wirtschaftlicher und sozialer Sicherheitsgarant künftiger Generationen ist."

Hier kann man getrost das Wort Pflicht durch das Wort Interesse ersetzen. Es geht um Härte bei der Erhaltung der Stabilität des Euro, bei der Eindämmung des Defizits, der Senkung der Staatsverschuldung und des Ausbaus der sozialen Marktwirtschaft - das sind die Leitlinien für Deutschland und genauso für Europa. Für ein Europa allerdings, dessen Regierungen gegenwärtig nicht gerade viel Energie für ein solches Programm aufbringen können. Werden die politischen und anderen Turbulenzen in einigen europäischen Nachbarstaaten und die Gestaltungsschwäche der Regierungen in einigen anderen EU-Mitgliedsstaaten durch deutsche Führungsschwäche oder Führungsmängel noch befördert, könnten sich in der Tat jene Vorhersagen erfüllen, nach denen Europa und damit auch alle seine Mitgliedsstaaten ihren Einfluss auf die Weltpolitik bald eingebüßt haben werden.

Weltpolitik ist aber unter dem Vorzeichen der Globalisierung ein Arbeitsfeld und eine Aufgabe, die keineswegs nur den Großmächten zufällt. Jeder Staat ist in sie eingebunden, mal mehr, mal weniger, und keiner kann sich daraus herauslösen. Weltpolitik bedeutet mehr als nur die jeweilige Projektion eigener Interessen und Werte auf die Leinwand der internationalen Politik. Sie stellt den Staaten (und auch nichtstaatlichen Akteuren) vor allem die Aufgabe, für einen internationalen Ordnungsrahmen mit hoher Verbindlichkeit zu sorgen, um politische und wirtschaftliche Austauschprozesse im internationalen System zu sichern. Konflikte wie der NahOst-Konflikt, der Bürgerkrieg in Afghanistan und Teilen Pakistans oder das Aufkommen einer neuartigen Piraterie in Afrika und Asien sind nicht lokal einzudämmen, entwickeln ein beträchtliches Stör-Potential und bedrohen im schlimmsten Fall nicht nur die Menschen in der Region, sondern weltweit.

Die westlichen Staaten und andere, die an stabilen weltpolitischen Verhältnissen interessiert sind, haben bislang nicht viele Erfolge aufzuweisen. Die großen Erwartungen, die sich an Konzepte wie „humanitäre Interventionen“ „nationbuilding“ oder „Demokratieexport“ geknüpft haben, erwiesen sich in den letzten zehn Jahren als verfehlt.

Deutschland hat sich auf der weltpolitischen Bühne eher zurückgehalten. Der Anspruch auf einen Ständigen Sitz im UNO-Sicherheitsrat, den alle Bundesregierungen seit Mitte der 1990er Jahre erhoben haben, drückt immerhin aus, dass diese Zurückhaltung nicht Desinteresse bedeutet. Deutschland wird zwar auf absehbare Zeit dieses Ziel nicht erreichen, aber auf eine (meist) zurückhaltende Art waren und sind die 
Bundesregierungen dennoch auf der weltpolitischen Bühne präsent. Man denke etwa an die deutschen Versuche, eine planetarische Klimapolitik anzustoßen - wenngleich diese wie ein paar andere Unternehmungen der deutschen Politik auf der weltpolitischen Ebene nur mäßig erfolgreich waren.

Deutsche Zurückhaltung in der Weltpolitik ist insgesamt sinnvoll. Aber es ist Ausdruck eines deutschen Führungsmangels, dass es bislang so wenig gelungen ist, die EU als weltpolitischen Akteur in Szene zu setzen. Selbstverständlich ist das schwierig, weil traditionsreiche Nationalstaaten wie Frankreich oder Großbritannien dabei nur ungern mitziehen. Aber da im Grunde alle Regierungen wissen, dass es keine Alternative dazu gibt, wenn Europa auf der Weltbühne entscheidend mitspielen will, sollte es ein europapolitischer Schwerpunkt der Bundesregierung sein, den weltpolitischen Akteurs-Status der EU weiter kräftig zu befördern.

\section{Ausblick}

Die ersten eineinhalb Jahre der schwarz-gelben Koalitionsregierung waren eine Zeit der vollmundigen Ankündigungen, simulierten Visionen und hektischen Umsteuerungen. Manches davon ist auf unerwartete Einflüsse von außen zurückzuführen. Ganz deutlich sind aber die personellen Schwächen der Regierung unter Kanzlerin Angela Merkel hervorgetreten, besonders in den beiden kleineren Koalitionspartnern. In der öffentlichen Wahrnehmung überwiegt Kritik. Medien, die dieser Regierung eher distanziert gegenüberstehen, haben als eine der Ursachen für das schwache Auftreten der Regierung mangelnde Führungskraft ausgemacht. Das lässt sich in der Tat nicht einfach vom Tisch wischen.

Allerdings spielt bei der Beurteilung von Regierungshandeln auch der Faktor Zeit eine nicht zu unterschätzende Rolle. Gerade in Bezug auf die Person der Bundeskanzlerin ist es schwierig, ihre Verlautbarungen und Entscheidungen sofort genau abzuwägen und entsprechend entweder als Ausdruck von Führungsschwäche oder gerade des Gegenteils zu bewerten. Das liegt daran, dass in der Politik viele Entscheidungen auf andere als nur die unmittelbar erkennbaren politischen Ziele ausgerichtet sind. Angela Merkels Rolle im Vorfeld der Wahl des Bundespräsidenten Christian Wulff, ihre Äußerungen zu dem Bestseller von Thilo Sarrazin („nicht hilfreich“) oder ihre Kehrtwende in Sachen Atomenergie, das sind nur ein paar Beispiele dafür, dass ihr Verhalten mit zeitlichem Abstand durchaus nicht einfach nur als Ausdruck von Führungsschwäche gesehen werden kann. Es könnte sogar sein, dass die groben Regierungsfehler der schwarzgelben Koalition aus weiterer Perspektive auch nicht mehr ins Gewicht fallen werden als die entsprechenden Fehlhandlungen anderer neu ins Amt gelangender Bundesregierungen. Wer erinnert sich heute noch z. B. an die zahlreichen Pannen in den ersten Monaten der sozial-liberalen Koalition 1969/70? Und selbst die enormen Fehler der rot-grünen Koalition 1998/99 deckt schon der Mantel des Vergessens zu.

Führungsprobleme und Führungsschwäche sind eine zu vielschichtige und zu ernsthafte Thematik, um sie so anzuge- hen wie SPIEGEL-Online. Die Herausforderungen im Innern, in Europa, auf der Ebene der Weltpolitik, denen sich Deutschland heute ausgesetzt sieht, sind so riesig, dass sie nur mit Entschiedenheit und Führungsstärke beantwortet werden können. Aber nur dann, wenn die Berliner Politik solche Führungsstärke entwickelt, wird sich einmal mehr der ebenso alte wie triviale Spruch bewahrheiten können: Nichts ist so erfolgreich wie der Erfolg.

\section{Literatur}

Gast, H. (2009). Was bedeutet, politische Führung'? Eine Übersicht über unterschiedliche Definitionen aus interdisziplinärer Perspektive und ein Vorschlag zur systematischen Analyse des Gegenstandes anhand des Strukturfunktionalismus. Zeitschrift für Politikberatung, 2(2), 211-229.

Geis, M. (2010). Autorität. Mit Nüchternheit und Vorsicht ist Angela Merkel groß geworden. Jetzt wird ihre Stärke zur Schwäche. Und sie kann es kaum glauben. Die Zeit. Verfügbar unter http:// www.zeit.de/2010/28/Merkel [10. Juli 2010]

Grasselt, N. \& Korte, K.-R. (2007). Führung in Politik und Wirtschaft. Instrumente, Stile und Techniken. Wiesbaden: VS-Verlag.

Helms L. (2010). Leadership-Forschung als Demokratiewissenschaft. Aus Politik und Zeitgeschichte, B2-3, 3-8.

Helms, L. (2009). Politische Führung in der Demokratie. Möglichkeiten und Grenzen der vergleichenden Forschung. Zeitschrift für Politik, 56, 375-396.

Meyer, M. (2001). Mediokratie. Die Kolonisierung der Politik in den Medien. Frankfurt/M.: Suhrkamp.

Purini, A. P. (2010). Dies ist die Stunde von Angela Merkel. Die Zeit. Verfügbar unter http://www.zeit.de/2010/35/P-op-Europa-Merkel [26. August 2010]

Ritzi, C. \& Schaal, G.S. (2010). Politische Führung in der ,Postdemokratie. Aus Politik und Zeitgeschichte, B2-3, 9-15.

Schmidt, H. (2010). Nur keine Anmaßung. Deutschland darf der Versuchung zu Alleingängen in Europa nicht nachgeben. Die Zeit. Verfügbar unter http://www.zeit.de/2010/28/Point-Alpha-Preis [11. Juli 2010]

Sebaldt, M. \& Gast, H. (Hrsg.) (2010). Politische Führung in westlichen Regierungssystemen. Theorie und Praxis im internationalen Vergleich. Wiesbaden: VS-Verlag.

Schuett-Wetschky, E. (2008). Richtlinienkompetenz (hierarchische Führung) oder demokratische politische Führung? Antwort an Everhard Holtmann. In E. v. Holtmann \& W. J. Patzelt (Hrsg.), Führen Regierungen tatsächlich? Zur Praxis gouvernementalen Handelns (S. 85-97). Wiesbaden: VS-Verlag.

Spiegel Online (2010). Der nächste Neustart kommt bestimmt. Verfügbar unter http://www.spiegel.de/politik/deutschland/o,1518,704124,0o.html [1. Juli 2010]

von Bredow, W. (2010). Bundeswehr-Reform. Kleine Schritte und ein großer Sprung. Zeitschrift für Staats- und Europawissenschaften, 8(3), 384-411.

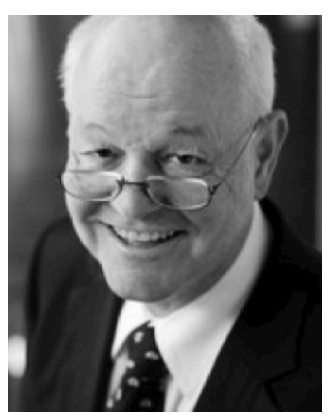

Prof. Dr. Dr. hc. Wilfried von Bredow hatte bis zu seiner Emeritierung 2009 einen Lehrstuhl für Politikwissenschaft an der Philipps-Universität Marburg inne. Derzeit ist er DAAD-Gastdozent am Centre of Comparative Literature und am Department of Political Science der Univerity of Toronto, Kanada. E-Mail:wvb@staff.uni-marburg.de. 